\title{
Programas bilingües en Educación Primaria: Valoraciones de docentes
}

\author{
María Lova Mellado \\ María José Bolarín Martínez \\ Mónica Porto Currás \\ Universidad de Murcia
}

Recibido: 4 de octubre de 2012 / Aceptado: 7 de marzo 2013

ISSN: $1697-7467$

\begin{abstract}
RESUMEN: Este artículo es fruto de una investigación que tenía como objetivo conocer las valoraciones de docentes de Educación Primaria del Programa Bilingüe de la Región de Murcia sobre las siguientes dimensiones: formación y coordinación del profesorado, metodología y evaluación, percepciones de los resultados, recursos e incentivos y familias. La metodología de investigación empleada fue de corte cualitativo, utilizando como instrumento la entrevista abierta a 14 profesores. Los principales resultados obtenidos señalan que se produjo: un incremento de coordinación del profesorado, una mejora de la imagen del colegio, actitudes positivas de los alumnos hacia la lengua inglesa y un aumento percibido del nivel de inglés de los escolares.
\end{abstract}

Palabras clave: Educación Primaria, bilingüismo, profesorado, formación y metodología.

\section{Bilingual Programs in Primary Education: Teachers'opinions}

\begin{abstract}
This article is the result of a research aimed to know the ratings of teachers of the Primary Bilingual Programme in the Region of Murcia, focused on the following dimensions: teacher training, coordination of the teaching staff, methodology and assessment, perceptions of results, resources and incentives and families. The research methodology applied was qualitative, using as a tool open interviews to fourteen teachers. The main results show: increase in coordination of teachers, improvement the image of the school, positive attitudes of students towards English language and a perceived increase in the English level of students.
\end{abstract}

Keywords: Primary Education, bilingualism, teachers, teaching and methodology.

\section{INTRODUCCIÓN}

La normativa que reguló la enseñanza de la lengua extranjera en España produjo unos resultados muy negativos (Plan de Fomento del Plurilingüismo), de ahí que hasta la década de los 80, exceptuando el caso de cinco comunidades que tienen dos lenguas oficiales, España se haya caracterizado por su monolingüismo. Según el último informe de la Organización de Cooperación y Desarrollo Económico (OCDE) los alumnos de Educación Secundaria de España poseen un dominio de las lenguas extranjeras muy por debajo de la media Europea 
(Rodríguez, 2010). Parece evidente, que ser ciudadano de hoy conlleva la necesidad de poseer un buen dominio de, al menos, dos idiomas además de la lengua materna; por tanto, si se quiere preparar a personas competentes para vivir en una comunidad multilingüe, la educación bilingüe es, sin duda, una de las mejores alternativas (Sánchez y Rodríguez, 1986). De ahí, el interés cada vez más creciente acerca de los temas concernientes al aprendizaje y enseñanza de una segunda lengua.

En esta línea, el sistema educativo español está implementando medidas para mejorar las competencias lingüísticas del alumnado. La Ley Orgánica de Educación 2/2006 establece como uno de los principios y fines fundamentales la capacitación del alumnado para la comunicación en la lengua oficial y cooficial, si la hubiere, y en una o más lenguas extranjeras (artículo 2.j) y propone que las Administraciones educativas promuevan la formación en lenguas extranjeras de todo el profesorado, independientemente de su especialidad, estableciendo programas específicos de formación en este ámbito (artículo 102.3). También, el artículo 17.f de la citada ley señala como uno de los objetivos de la Educación Primaria, la necesidad de adquirir en al menos una lengua extranjera, la competencia comunicativa básica que les permita expresar y comprender mensajes sencillos y desenvolverse en situaciones cotidianas.

Además, la Disposición Adicional Primera del Decreto 286/2007 de la Comunidad Autónoma de la Región de Murcia establece que la Consejería competente en materia de educación podrá autorizar que una parte de las áreas del currículo se imparta en lenguas extranjeras. A este respecto, esta Comunidad Autónoma puso en marcha la implantación del Programa Colegios Bilingües Región de Murcia, medida educativa adoptada por numerosos países europeos, con la finalidad de lograr un uso adecuado y simultáneo de la lengua castellana y de la lengua inglesa en la Educción Primaria, así como la adquisición de la competencia comunicativa que le permita desenvolverse con naturalidad en situaciones cotidianas.

Con la pretensión de conseguir dicho fin, los Programas Bilingües están basados en la integración de los contenidos y el aprendizaje de las lenguas extranjeras, donde los idiomas son utilizados como lenguas vehiculares mediante las que se adquieren los contenidos lingüísticos y académicos explicitados en el currículo (García, citado en Ramos, 2007b). Tratándose de un Programa con un enfoque metodológico que va más allá de la enseñanza de una lengua extranjera, parece necesario plantear desde la perspectiva docente, las necesidades reales que tienen los docentes adscritos a este programa desde su implantación en el curso escolar 2009/2010, así como las herramientas y conocimientos que son precisos desarrollar, para un desempeño eficaz de la actividad en las aulas bilingües.

En este artículo describiremos el fundamento teórico y marco normativo del Programa Bilingüe de la Región de Murcia. Posteriormente, expondremos el proceso de recogida de datos, así como de transcripción y análisis. Tras ello, presentaremos los resultados obtenidos con la finalidad de identificar las principales problemáticas surgidas en la implementación de este Programa, así como aquellos aspectos más específicos que por sus singulares características afectan de un modo especial al docente y que a su vez contribuyen al éxito de una enseñanza bilingüe de calidad. Por último, estableceremos las conclusiones extraídas de las opiniones de los docentes contenidas en las entrevistas. 


\section{Fundamentación teórica Y NORMATIVA DEL PROGRAMA BILINGÜE EN la Región de Murcia}

Atendiendo a la definición de bilingüismo de Siguan y Mackey (1986), quienes sostienen que los programas bilingües enseñan en dos lenguas, una de las cuales suele ser el idioma nativo de los alumnos, el objetivo de estos programas es que los estudiantes aprendan la segunda lengua sin olvidar la lengua materna, es decir, un bilingüismo aditivo (Baker, 1997) y no un bilingüismo substractivo en el que idioma nativo desaparezca. Por ello, los idiomas son utilizados como lenguas vehiculares de comunicación e instrucción en las asignaturas del currículo educativo, en lugar de ser meros objetos de estudio sin aplicación práctica inmediata (Ramos, 2009). En este sentido, y teniendo en cuenta, que aprender una lengua no es lo mismo que usar una lengua (González, 2005), cobra especial relevancia el hacer con y a través de la lengua, mediante intercambios comunicativos que simulen situaciones de la vida real, propiciando el uso auténtico de la segunda lengua. Se recomiendan tareas como diálogos, juegos, trabajos en parejas o en pequeños grupos y juegos de roles, entre otras actividades comunicativas, ya que dichas tareas provocan en el alumnado una necesidad de comunicarse y crear significados, condición esencial para lograr la competencia comunicativa (Benítez, citado en Corrales, 2009:159). En esta línea, es necesaria una metodología de trabajo que propicie el paso de la enseñanza del inglés a la enseñanza en inglés. Este paso supone la necesidad de considerar un currículo integrado que permita la adquisición tanto de los contenidos curriculares como de la lengua. Entendemos por currículo integrado, aquel que integra no sólo las lenguas enseñadas en un colegio sino también las áreas de conocimiento relacionadas con la enseñanza bilingüe. Este currículo plasmará las capacidades del alumnado en relación con la reflexión sobre las lenguas, la familiarización con las culturas y el uso de la comunicación para la adquisición de contenidos (Siguán, citado en Molero, 2011a:8). Asimismo, no debemos olvidar, como señala Halbach (2008), que la planificación de dicho currículo requiere un alto grado de coordinación entre los docentes adscritos al programa.

Podemos decir que el bilingüismo fomenta la creatividad y flexibilidad cognitiva al obligar a los alumnos a usar los recursos que poseen en los dos idiomas; además de beneficiarles metalingüística e intelectualmente, al exigirles utilizar y desarrollar más estrategias de comunicación y resolución de problemas (Cummins, 2002). Siguiendo a Pérez (2008), el desarrollo de estrategias de aprendizaje, en ocasiones no habituales para la edad de los escolares, implica una repercusión favorable tanto en las asignaturas impartidas en lengua materna como en la segunda lengua.

Como beneficio añadido, los escolares desarrollan actitudes positivas hacia otros idiomas y culturas (Soltero, citado en Ramos, 2007b). Cuando se enseña otra lengua se enseñan otros valores, la educación bilingüe enriquece las posibilidades de expresión y comunicación a la vez que incrementa la amplitud de miras, fomentando un espíritu abierto a la diversidad.

En la Región de Murcia, la Consejería de Educación, Formación y Empleo, pone en marcha en el año 2009, el programa Colegios Bilingües Español-Inglés. Actualmente, son 112 centros docentes de Educación Infantil y Primaria, tanto públicos como privados concertados, en los que se lleva a cabo el programa bilingüe.

Este programa pretende, según la Orden de 18 de abril de 2011, el logro de un uso adecuado y simultáneo de la lengua castellana y de la lengua inglesa en Educación Primaria, así como la adquisición de la competencia comunicativa suficiente para desenvolverse 
con naturalidad en situaciones cotidianas. Promoviendo la formación de alumnos, futuros ciudadanos, capacitados para enfrentarse con éxito a los retos que la sociedad europea, plural, competitiva, abierta y en constante evolución pueda depararles en un futuro no muy lejano.

Esta Orden especifica que se entiende por enseñanza bilingüe la que permite impartir, al menos, un cuarto del horario lectivo semanal en lengua inglesa. Indica que los centros seleccionados, además del horario dedicado al Idioma Extranjero: Inglés, deberán impartir en este idioma otras áreas hasta alcanzar el mencionado cuarto del horario semanal. Además, establece que dicha elección podrá ser llevada a cabo entre las siguientes áreas: Conocimiento del Medio Natural, Social y Cultural, Educación Artística y Educación Física.

Asimismo, menciona que la implantación del programa se iniciará en el primer curso de Educación Primaria de los colegios que resulten seleccionados a través de la correspondiente convocatoria, y se llevará a cabo de manera gradual, curso a curso, implantándose en la totalidad de unidades de cada nivel.

En lo relativo al profesorado, factor determinante en el éxito de los programas bilingües (Castro, 2002), la Orden regula que deberá poseer la titulación de diplomatura en Magisterio, especialidad Lengua Extranjera Inglés o licenciatura en Filología Inglesa o, en su caso, profesorado habilitado para desempeñar puestos de trabajo en el área de Lengua Extranjera Inglés o la titulación de diplomatura en Magisterio, independientemente de la especialidad, y el nivel B2 de competencia comunicativa indicado en el Marco Común Europeo de Referencia, que podrá ser acreditado por una Escuela Oficial de Idiomas o cualquier otra institución de reconocimiento oficial en el espacio europeo.

También, señala como condiciones obligatorias que los docentes sean funcionarios de carrera con destino definitivo en el centro solicitante $y$, sin menoscabo de sus derechos como funcionario, no participar en el concurso de trasladados durante, al menos, dos cursos escolares. El artículo 12 de la citada Orden, expresa que todos los maestros contraen la obligación de asistir a las actividades programadas dentro del programa de actualización del profesorado en centros adscritos al Programa Colegios Bilingües Región de Murcia que consistirá en un curso de formación inicial sobre contenidos metodológicos específicos para la enseñanza bilingüe y la metodología AICLE con una duración mínima de 150 horas.

La citada Orden también establece que los centros adscritos al programa dispondrán de Auxiliares de Conversación de lengua inglesa, con el fin de proveer refuerzo lingüístico tanto en la asignatura de Lengua Extranjera Inglés como en las impartidas en este idioma (Ramos, 2007b), pero no especifica los requisitos ni las funciones de estos profesionales.

En cuanto a las tareas que el profesorado participante en el programa deberá llevar a cabo, la Orden indica: adaptar el currículo de su área incorporando aspectos lingüísticos, culturales, sociales y educativos relativos a países de habla inglesa; elaborar los materiales didácticos necesarios en coordinación con el resto del profesorado, especialmente el de la lengua extranjera objeto del programa y, en su caso, con el auxiliar de conversación; e impartir la docencia directa en lengua inglesa a los grupos de alumnos en los que se implante el Programa Colegios Bilingües Región de Murcia durante al menos dos cursos escolares.

Si bien, la Orden pone de manifiesto, la importancia que la coordinación tiene en los Programas Bilingües, al indicar que los profesores han de trabajar con sus compañeros y con los auxiliares de conversación en la creación de materiales en los dos idiomas, no define ni concreta a nivel legal tiempos para la realización de estas tareas. Tampoco contempla una 
reducción horaria para el docente que desempeña la función de coordinador del programa, a pesar de ser la persona encargada tanto de velar por el buen funcionamiento interno del programa, como de establecer vínculos comunicativos e interactivos con profesorado de otros centros adscritos al programa.

Es evidente que el profesorado involucrado en el Programa Bilingüe ha de contar con una formación no sólo en conocimientos sobre teorías y métodos de aprendizaje de idiomas, sino también en una mejora de las técnicas que potencian las destrezas básicas de los alumnos en la lengua inglesa con el fin de estar capacitado para enseñar los contenidos de las materias curriculares a través de una segunda lengua.

En este sentido, la enseñanza bilingüe ha supuesto un nuevo reto educativo para todos los docentes participantes en estos programas, ya que esta enseñanza implica cambios metodológicos, curriculares y organizativos conducentes a la consecución de metas más allá de lo lingüístico.

Podríamos pensar que en la educación bilingüe existe una metodología apropiada y establecida, pero no es así, debido a que la educación bilingüe se presenta como un concepto nebuloso que engloba numerosos enfoques de enseñanza y que la metodología adecuada para un proyecto puede no serlo para otros (Halbach, 2008). No obstante, es necesario atender a la integración de la enseñanza de contenido y lengua que ha de realizarse respetando la unidad existente entre ellas, con el fin de que los escolares entiendan los conocimientos adquiridos como un todo, y no como aprendizajes aislados e independientes. La integración no tiene que estar limitada a las materias impartidas en lengua inglesa, sino que puede hacerse extensible a todas las asignaturas, coordinándose en torno a un tema concreto, y logrando así una mayor sensación de unidad en el aprendizaje de los alumnos. Obviamente, ello requiere la coordinación entre todo el profesorado con la finalidad de intercambiar materiales y actividades, buscar textos cercanos a los niños y diseñar actividades conjuntas.

Del mismo modo, la cooperación entre alumnos resulta muy beneficiosa y se hace más necesaria cuando la materia se imparte en L2, ya que en estas clases los alumnos suelen tener niveles lingüísticos dispares. El aprendizaje cooperativo ocasiona la interacción entre los alumnos con el fin de generar conocimiento y garantizar que se sientan responsables de su aprendizaje individual y colectivo (Trujillo, 2007). Por ello, el trabajo cooperativo es fuente de conocimiento y motor de desarrollo interpersonal.

En definitiva, todas las propuestas metodológicas son esenciales para afrontar este reto: una enseñanza bilingüe de calidad. Por ello, la mayoría de las investigaciones existentes acerca de los proyectos educativos bilingües han estado focalizadas en los efectos que tienen dichos programas sobre la producción lingüística de los estudiantes (Daniel and Nerlich, y Zydatiss, citados en Fernández et al., 2005), el desarrollo de su conciencia intercultural (Fehling, 2002), sus beneficios (Baker, 1997; Cummins, 2002; Howard and Christian, 1997; Howard, Christian and Genesse, 2004 y Soltero, citado en Ramos, 2007b), o en los problemas de la enseñanza bilingüe como la adaptación y creación de materiales didácticos (Krück and Loeser, citado en Laorden y Peñafiel, 2010).

Centrándonos en España, es importante expresar que el hecho de que cuente con una realidad multilingüe en el propio territorio nacional ha ocasionado que muchos de los esfuerzos se hayan basado, principalmente, en cómo integrar el castellano con las lenguas oficiales de las comunidades autónomas correspondientes (Laorden y Peñafiel, 2010; Siguán, citado en Molero, 2011a; Olaziregi, 1994; Urrutia et al., 1998). También, es importante resaltar los 
estudios que se han llevando a cabo sobre la enseñanza de un tercer idioma en comunidades autónomas bilingües, originando modelos educativos trilingües (Lasagabaster, Ruiz de Zarobe y Torres-Guzmán and Etxeberría, citados en Ramos, 2007a y Cenoz and Jessner, 2000).

Asimismo, los estudios llevados a cabo en diversas Comunidades Autónomas de este país, acerca de la enseñanza bilingüe inglés-español en la etapa de Educación Primaria han estado centrados en: metodologías adecuadas para este tipo de educación (Halbach, 2008; Molero, 2011a), propuestas AICLE (García Jiménez, García Pellicer y Yuste, 2012; Fernández, 2008; Fernández, 2009; Ramos, 2009; Ramos y Ruiz 2011), percepción de los equipos directivos (Laorden y Peñafiel, 2010), así como opiniones de los alumnos sobre estos programas (Ramos, 2007a). Sin embargo, los estudios referidos al profesorado han estado focalizados principalmente en: propuestas para su formación en la enseñanza bilingüe (Ramos, 2007b), orientaciones sobre la enseñanza bilingüe en distintas asignaturas (Cancelas y Cancelas, 2009; Fernández, 2009 y Molero, 2011b) y en sus expectativas antes de iniciar el proyecto bilingüe (Fernández et al., 2005). Por lo que, los existentes acerca de las percepciones de los maestros de Educación Primaria participantes en estos programas, tema del que versa este artículo, han sido escasos, destacando a Pavón and Rubio (2010) basado en las preocupaciones e incertidumbres de los maestros acerca principalmente de la metodología y la implementación del currículo integrado; Travé (2011) centrado en el concepto de bilingüismo y enseñanza bilingüe, experiencias bilingües y proyecto bilingüe; y a Lorenzo, Casal, Moore y Alfonso (2009), quienes destinan una sección de su estudio de la situación de la Red de Centros Bilingües en Andalucía a las valoraciones que los maestros implicados en los programas bilingües poseen en torno a diferentes aspectos: formación metodológica y lingüística, profesorado nativo, coordinación docente, recursos y reducción horaria, entre otros.

\section{Metodología}

Nuestro objetivo general era conocer la valoración de los docentes adscritos al Programa Bilingüe de la Región de Murcia desde su implantación en el curso escolar 2009/2010, en relación a diferentes dimensiones: formación del profesorado, coordinación de los docentes, metodología de enseñanza y evaluación, percepciones de los resultados, recursos e incentivos y participación de los padres.

La metodología de investigación empleada fue de corte cualitativo, estudio descriptivoexploratorio, utilizando como instrumento la entrevista abierta o en profundidad con un nivel de directividad medio-bajo y, semiestructurada en cuanto a que se plantean unos interrogantes de carácter abierto con el objeto de que expresen libremente sus ideas, creencias, opiniones, valoraciones (Rodríguez, Gil y García, 1996; Tójar, 2006). Hemos optado por utilizar la entrevista porque ofrece la posibilidad al entrevistado de hablar sobre la conveniencia o no de una pregunta, corregirla, hacer alguna puntualización o responder de la forma que estimen conveniente (Agar, citado en Rodríguez, Gil y García, 1996), haciéndolos participantes activos de la entrevista y por tanto, colaboradores imprescindibles en nuestra investigación.

Las entrevistas fueron realizadas a catorce maestros de ocho centros públicos de Educación Infantil y Primaria, de los ciento doce participantes, distribuidos por la Región de Murcia. Aunque la investigación se realiza como un estudio exploratorio, en aras de buscar 
una muestra heterogénea hemos optado por utilizar como variable de selección los años de participación en el programa, por ello, el número de años de participación oscila entre los tres años, periodo de tiempo que este programa se puso en marcha, hasta docentes que se han incorporado en este curso.

Tabla 1. Perfil de los docentes adscritos al Programa Bilingüe.

\begin{tabular}{|c|c|c|c|c|c|c|c|c|c|}
\hline & Edad & Sexo & $\begin{array}{l}\text { Docencia } \\
\text { en E.P } \\
\text { (Años) }\end{array}$ & $\begin{array}{c}\text { Docencia en P. B } \\
\text { (Años) }\end{array}$ & $\begin{array}{l}\text { Años } \\
\text { centro } \\
\text { en PB }\end{array}$ & Titulación & $\begin{array}{l}\text { Formación } \\
\text { complementaria } \\
\text { (inglés) }\end{array}$ & $\begin{array}{l}\text { Asignaturas } \\
\text { imparten }\end{array}$ & Nivel \\
\hline E1 & 35 & $\mathrm{M}$ & 8 & 1 & 2 & $\begin{array}{c}\text { LE } \\
\text { Francés }\end{array}$ & $\begin{array}{c}\text { N. } \\
\text { Avanzado }\end{array}$ & $\begin{array}{l}\text { C.Medio } \\
\text { Inglés. }\end{array}$ & $1 .^{\circ}$ \\
\hline E2 & 44 & $\mathrm{M}$ & 21 & 2 & 2 & $\begin{array}{l}\text { L.E. } \\
\text { Inglés. }\end{array}$ & $\begin{array}{c}\text { N. } \\
\text { Avanzado }\end{array}$ & $\begin{array}{l}\text { C.Medio } \\
\text { Inglés. }\end{array}$ & $2 .^{\circ}$ \\
\hline E3 & 37 & $\mathrm{M}$ & 7 & 2 & 2 & $\begin{array}{l}\text { L.E. } \\
\text { Inglés. }\end{array}$ & & $\begin{array}{l}\text { C.Medio } \\
\text { Inglés. }\end{array}$ & $2 .^{\circ}{ }^{\circ}$ \\
\hline E4 & 43 & $\mathrm{M}$ & 20 & 2 & 2 & $\begin{array}{l}\text { E.Es } \\
\text { pecial } \\
\text { Peda } \\
\text { gogía }\end{array}$ & $\begin{array}{c}\text { N. } \\
\text { Avanzado }\end{array}$ & $\begin{array}{l}\text { C.Medio } \\
\text { Inglés. } \\
\text { E.Artística. }\end{array}$ & $\begin{array}{l}1 .^{\circ} \\
2 .{ }^{\circ}\end{array}$ \\
\hline E5 & 54 & $\mathrm{M}$ & 21 & 2 & 2 & $\begin{array}{l}\text { L.E. } \\
\text { Inglés. } \\
\text { Filolo } \\
\text { gía Inglesa }\end{array}$ & & $\begin{array}{l}\text { C.Medio } \\
\text { Inglés. } \\
\text { E. Artística }\end{array}$ & $2 .^{\circ}{ }^{\circ}$ \\
\hline E6 & 52 & $\mathrm{M}$ & 17 & 2 & 2 & $\begin{array}{l}\text { L.E. Inglés } \\
\text { y Francés }\end{array}$ & $\begin{array}{c}\mathrm{N} . \\
\text { Intermedio }\end{array}$ & $\begin{array}{l}\text { C.Medio } \\
\text { E.Artística } \\
\text { Inglés. }\end{array}$ & $2 .^{\circ}$ \\
\hline E7 & 37 & M & 5 & 1 & 1 & $\begin{array}{l}\text { L.E } \\
\text { Inglés. }\end{array}$ & $\begin{array}{c}\text { N. } \\
\text { Avanzado }\end{array}$ & $\begin{array}{l}\text { C.Medio } \\
\text { Inglés. }\end{array}$ & $1 .^{\circ}$ \\
\hline E8 & 48 & $\mathrm{M}$ & 26 & 3 & 3 & $\begin{array}{l}\text { L.E. } \\
\text { Inglés. }\end{array}$ & $\begin{array}{c}\text { N. } \\
\text { Avanzado }\end{array}$ & $\begin{array}{l}\text { C.Medio } \\
\text { E.Artística. } \\
\text { Inglés. }\end{array}$ & $3 .^{\circ}$ \\
\hline E9 & 32 & $\mathrm{H}$ & 5 & 1 & 3 & $\begin{array}{l}\text { L.E. } \\
\text { Inglés. }\end{array}$ & $\begin{array}{c}\text { N. } \\
\text { Avanzado }\end{array}$ & $\begin{array}{l}\text { C.Medio } \\
\text { E.Artística } \\
\text { Inglés. }\end{array}$ & $1 .^{\circ}$ \\
\hline E10 & 38 & $\mathrm{M}$ & 5 & 1 & 1 & L.E. Inglés & $\begin{array}{c}\text { N. } \\
\text { Avanzado }\end{array}$ & $\begin{array}{l}\text { C.Medio. } \\
\text { E.Artísica. } \\
\text { Inglés. }\end{array}$ & $1 .^{\circ}$ \\
\hline E11 & 38 & M & 13 & 1 & 1 & L.E. Inglés & $\begin{array}{c}\mathrm{N} . \\
\text { Avanzado }\end{array}$ & E. Artística. & $1 .^{\circ}$ \\
\hline E12 & 35 & $\mathrm{H}$ & 3 & 1 & 3 & $\begin{array}{c}\text { L.E. } \\
\text { Inglés } \\
\text { E.Física. }\end{array}$ & N. Intermedio & $\begin{array}{l}\text { C.Medio } \\
\text { E.Artística } \\
\text { Inglés. }\end{array}$ & $1 .^{\circ}$ \\
\hline E13 & 54 & M & 32 & 2 & 2 & $\begin{array}{l}\text { L.E. } \\
\text { Inglés. }\end{array}$ & & $\begin{array}{l}\text { C.Medio } \\
\text { E.Artística } \\
\text { Inglés }\end{array}$ & $2 .^{\circ}$ \\
\hline E14 & 34 & $\mathrm{H}$ & 11 & 2 & 2 & $\begin{array}{l}\text { L.E. } \\
\text { Inglés }\end{array}$ & N. Intermedio. & $\begin{array}{l}\text { C.Medio } \\
\text { Inglés }\end{array}$ & $1 .^{\circ}{ }^{\circ}$ \\
\hline
\end{tabular}

Como puede observarse en la tabla 1, doce entrevistados poseen la Diplomatura de Magisterio de Lengua Extranjera Inglés, y los otros dos tienen otras especialidades de Magisterio. El periodo de tiempo que estos maestros están impartiendo docencia en Educación Primaria es muy diverso, oscilando desde tres años hasta treinta y dos años. Siete centros han optado por impartir en lengua inglesa las asignaturas de Conocimiento del Medio Natural, Social y Cultural y Educación Artística en primero, segundo y tercer curso, excepto uno que sólo imparte Conocimiento del Medio. Además, ninguno de los entrevistados es tutor de un curso bilingüe. 
Para llevar a cabo estas entrevistas, se utilizó un guión orientativo, si bien fue adaptándose al ritmo y respuestas de los entrevistados, eliminando en cada caso aquellas preguntas que no procedían e incorporando de manera flexible las que fueran necesarias para centrar la conversación en el propósito de la misma. Las entrevistas se realizaron durante los meses de Febrero y Marzo de 2012, se grabaron y se transcribieron. Una vez transcrita la información recogida de las entrevistas, se procedió a su análisis cualitativo. Siguiendo el criterio temático (Rodríguez, Gil y García, 1996), la información fue agrupada en torno a categorías. Dichas categorías pueden considerarse como un mapa de significados que reflejan fielmente la estructura del contenido de las entrevistas y nos permite interpretar las valoraciones de los docentes en relación al problema de investigación.

\section{Resultados. VAloraciones de los docentes adscritos Al PRograma BILINGÜE DE LA REGIÓN DE MURCIA}

A nivel general podemos destacar que los resultados de las entrevistas al profesorado ofrecen una panorámica de la puesta en práctica del Programa bilingüe. Sin duda son numerosas las dificultades a las que se enfrenta, pero la satisfacción prácticamente unánime de los profesores entrevistados invita al optimismo. Los efectos de la enseñanza bilingüe en el alumnado y en el centro son muy positivos para estos docentes: propicia una mejora significativa en el proceso de enseñanza-aprendizaje, aumento de la imagen del colegio, aumento de la calidad de la formación.

A continuación, presentamos un análisis pormenorizado de las valoraciones proporcionadas por los docentes entrevistados.

\subsection{Formación del profesorado}

Doce docentes realizaron el curso de metodología AICLE propuesto por la Consejería, y su satisfacción es alta puesto que piensan que la formación fue útil y necesaria. Sin embargo, muestran su descontento con aspectos relacionados con la planificación de dicho curso, concretamente, con las fechas seleccionadas para llevarlo a cabo y con la duración del curso.

Además, cabe mencionar que el hecho de que dos de los seis maestros que se han incorporado al programa en este curso escolar no hayan recibido esta formación, parece indicar la necesidad de mejorar los criterios de selección de docentes para la realización de este curso, así como un aumento de las plazas ofertadas.

Respecto a la formación continua, cuatro de los siete docentes que reciben cursos en inglés consideran que se podría mejorar, y aunque todos los participantes están interesados en asistir a las jornadas de seguimiento, sólo dos las están realizando actualmente, por lo que podemos indicar que existen necesidades formativas no cubiertas que repercuten negativamente en la puesta en práctica del Programa Bilingüe.

\subsection{Coordinación del profesorado}

Todos los entrevistados se reúnen con los compañeros participantes en el programa en alguna hora libre, puesto que no existen unas horas establecidas oficialmente para la coor- 
dinación de los profesores bilingües. Ahora bien, podemos resaltar que cuanto mayor es el número de profesores implicados, la coordinación se lleva a cabo principalmente con los docentes del mismo nivel y más esporádicamente con el resto de maestros de otros niveles. Otros profesionales con los que se coordinan los entrevistados, aunque no todos, son con los tutores de los cursos que imparten clase y con el resto de maestros de Lengua Extranjera del centro. Afirman que se coordinan con los auxiliares de conversación, principalmente mediante contactos informales, ya que consideran que su labor es fundamental tanto para los alumnos como para ellos mismos. En general, todos los docentes consideran que el trabajo de coordinación que llevan a cabo con el programa es mayor del que realizaban antes.

Por otro lado, todos los entrevistados tienen contacto con algunos colegios adscritos al programa, por lo que excepto dos, todos afirman que conocen un poco cómo llevan a cabo el programa en otros centros educativos.

\subsection{Enseñanza y Programa Bilingüe}

La estructura utilizada para la organización de los contenidos impartidos en L2 no difiere de la empleada antes de incorporarse al programa. Sin embargo, consideran que los contenidos de las asignaturas impartidas en L2 son enseñados de forma práctica y manipulativa, empleando la observación y experimentación.

En general, todos los entrevistados han incorporado aspectos socioculturales relativos a países de habla inglesa en las asignaturas impartidas en L2, abordados principalmente por los auxiliares de conversación.

Asimismo, todos los participantes que imparten lengua extranjera en cursos bilingües apoyan los contenidos de las materias impartidas en L2, por ejemplo introduciendo el vocabulario que van a utilizar en Conocimiento del Medio. Además, casi todos complementan las dos o tres asignaturas que imparten en inglés, por lo que abordan los contenidos de una temática concreta al mismo tiempo, excepto un caso que intenta tratarlos siempre antes en una asignatura y después en la otra con la finalidad de que les sirva de repaso.

Estos docentes no poseen un sistema de evaluación específico, pero todos creen que los criterios de evaluación difieren de los empleados habitualmente en las asignaturas impartidas en L2, puesto que han incorporado la valoración de la expresión y comprensión oral en inglés $\mathrm{y}$, en menor medida, la comprensión y expresión escrita.

Respecto a las dificultades que encuentran en el desarrollo de sus clases, estos entrevistados destacaron las siguientes: la incorporación de los niños a primer curso de Educación Primaria, la búsqueda de diversas formas de abordar los contenidos en lengua inglesa, ya que de por sí algunos conocimientos son complejos hasta en su lengua materna, no tener un aula bilingüe y la adaptación de niños con necesidades educativas especiales (NEE) a las clases bilingües. Además, todos los participantes destacaron la complejidad que va a conllevar encauzar a los escolares que han realizado cursos no bilingües y que al repetir se incorporarán a aulas bilingües, así como la adaptación de los escolares con NEE e inmigrantes.

\subsection{Percepciones sobre los resultados obtenidos}

Todos los docentes creen que los alumnos se desenvolverán con naturalidad al terminar la Educación Primaria, aunque resaltaron que si la Consejería se implicara más en esta iniciativa podrían lograrse mejores resultados. 
Además, casi todos los entrevistados, excepto uno, consideran que la imagen del colegio es más positiva con la implantación del programa, al haber incrementado tanto su prestigio como la calidad de la formación ofertada, puesto que preparan alumnos más competentes para hacer frente a esta sociedad globalizada.

En cuanto a los alumnos, todos los participantes expresaron que muestran actitudes positivas hacia el inglés. Sin embargo, en relación al interés de los escolares por aspectos socioculturales de los países de habla inglesa, mientras nueve docentes destacaron que ha aumentado, otros cinco consideran que tienen el mismo interés.

\subsection{Recursos e incentivos}

La mayoría de los entrevistados (diez) expresaron que los centros bilingües han sido dotados de muy pocos recursos para este tipo de docencia, por lo que son ellos quienes elaboran los materiales, e incluso tres destacaron que adquieren algunos costeándolos con su dinero. Además, sólo dos de los entrevistados poseen un aula bilingüe.

En relación a los recursos tecnológicos, once entrevistados expresaron que son insuficientes para afrontar eficazmente este tipo de docencia y siete docentes manifestaron que no poseen pizarras digitales en todas las aulas, por lo que cada vez que quieren usarla tienen que desplazarse a otras salas.

Además, casi la totalidad de los docentes perciben mayor la carga de trabajo con el Programa Bilingüe. Esto, unido al hecho de no recibir incentivos por participar en el programa, ni ningún tipo de reconocimiento provoca una situación de insatisfacción con la Consejería, destacando que su único aliciente actualmente es los progresos que perciben en los alumnos.

\subsection{Familias}

Todos los entrevistados expresaron que los progenitores están satisfechos con la incorporación de los centros educativos al Programa Bilingüe. Las diferencias en cuanto al interés de los padres y su implicación en la educación de sus hijos oscilan entre interés alto y asistencia las tutorías por la mayoría de las familias (declaración de doce maestros), e interés muy alto y que la totalidad de los familias asisten a dichas tutorías (dos maestros). Asimismo, en cuanto al papel que se les otorga a los padres con el programa, se perciben discrepancias entre los docentes puesto que si bien diez maestros indicaron que difiere del que se le concedía antes ya que ahora se les solicita una mayor implicación en la educación de sus hijos, cuatro señalaron que es el mismo.

\section{Conclusiones y propuestas de mejora}

Aunque la mayoría de docentes acreditan tener formación lingüística y metodológica para impartir docencia en este programa, consideramos prioritario incrementar las posibilidades de formación continua de estos maestros tanto en conocimientos sobre teorías y métodos de aprendizaje de idiomas, como en una mejora de las destrezas lingüísticas ya que 
algunos entrevistados afirmaron desconocer algunos vocablos específicos de conocimiento del medio en inglés, como constataron Lorenzo, Casal, Moore y Alfonso, 2009. También, parece apropiado que los futuros docentes reciban formación intensiva en inglés durante todas sus etapas formativas.

Hemos visto que la coordinación es útil e imprescindible en la implementación de un Programa Bilingüe para la calidad de su docencia. Sin embargo, hemos constatado que en la práctica no se contemplan tiempos a nivel legal para ello, suponiendo un mayor esfuerzo desde el propio profesorado para que la coordinación sea una realidad. Además, la coordinación debe ir más allá de los centros, aumentando el intercambio de experiencias con otros colegios, con la finalidad de que este constituya una fuente de formación para el profesorado, principalmente para los docentes que se incorporan por primera vez al programa.

Por otro lado, los docentes afirman que uno de los cambios que la adscripción de los colegios al Programa Bilingüe ha propiciado en la docencia de la enseñanza de la lengua extranjera en Educación Primaria es la relevancia de las habilidades comunicativas al otorgarles más valor, resultado constatado también por algunos docentes en la investigación de Fernández et al. (2005) y de Travé (2011). De ahí que los escolares al finalizar esta etapa logren desenvolverse mejor en este idioma. Otra de las repercusiones que señalan es un cambio metodológico, aunque no se puede generalizar, para la implementación de la enseñanza bilingüe, puesto que consideran necesario enseñar los contenidos de forma práctica y manipulativa, enfatizando su comprensión. Este cambio metodológico está reflejado en Lorenzo, Casal, Moore y Alfonso (2009) y en Pavón and Rubio (2010), estos últimos lo plantearon como un reto al que se enfrentaban los docentes involucrados en la enseñanza bilingüe.

En general, todos los docentes consideran que el centro ha mejorado con la implantación del programa en aspectos como mejora del prestigio, resultado constatado por Travé (2011), y del nivel de inglés de los alumnos, expuesto en Lorenzo, Casal, Moore y Alfonso (2009), y algunos también resaltan un aumento de matrículas en el centro así como un incremento de la coordinación y motivación entre el profesorado, resultados que coinciden con los expuestos por Laorden y Peñafiel (2010).

Sin embargo, la carga de trabajo de estos profesores ha aumentado significativamente con esta iniciativa, por lo que sería necesario que la Administración les concediera una reducción horaria. A su vez, estos entrevistados no reciben incentivos ni ningún otro tipo de reconocimiento por participar en el Programa Bilingüe, de ahí la diversidad de opiniones existentes acerca del tiempo que tienen previsto continuar impartiendo docencia en este programa.

No obstante, una de las motivaciones, junto con los progresos de los alumnos, que anima a estos maestros a seguir impartiendo docencia en el Programa Bilingüe es el apoyo recibido tanto por el equipo directivo como por toda la comunidad educativa, resultado expuesto en Lorenzo, Casal, Moore y Alfonso (2009), contradiciendo pues los obtenidos en el estudio de Laorden y Peñafiel (2010) quienes afirmaron que casi la mitad de los participantes percibieron resistencia al programa. Al contrario que este resultado, todos los maestros entrevistados afirmaron que las relaciones interpersonales entre los docentes participantes en el programa y el resto de profesores no se han debilitado.

Los resultados de este estudio coinciden con los señalados por Howard, Sugarman and Christian (2003); Lasagabaster (2001) y Lorenzo, Casal, Moore y Alfonso (2009) en la alta valoración de los padres respecto a la adscripción de los centros educativos al Programa 
Bilingüe. Asimismo, respecto a la incertidumbre mostrada por los maestros acerca del papel de los padres antes de implantarse el programa según Fernández et al. (2005), los resultados de nuestro estudio indican que el papel de los progenitores con el programa difiere del que se le concedía antes, destacando una mayor implicación en la educación de sus hijos, resultado reflejado también en Laorden y Peñafiel (2010).

Teniendo en cuenta los resultados de este estudio, consideramos que sería fundamental prestar atención a todas las necesidades que están surgiendo en la puesta en marcha del Programa Bilingüe de la Región de Murcia, con la finalidad de dar respuesta a todas ellas y garantizar la calidad y el éxito de estos programas.

En este sentido, establecemos algunas propuestas con la finalidad de contribuir a una mejora en la implementación del Programa Bilingüe de la Región de Murcia:

En relación a la formación del profesorado:

- Ampliación del número de plazas para la realización del curso AICLE, mejora de los criterios de selección de maestros para llevarlo a cabo, así como perfeccionamiento en la planificación de este curso, en cuanto al tiempo y a la forma.

- Mejora de los cursos de formación continua destinados al profesorado participante, tanto los relativos a metodología específica como los de competencia lingüística.

- Realización de cursos o seminarios dirigidos a todos los profesores implicados en estos programas, con la finalidad de compartir e intercambiar experiencias y materiales y contribuir a la resolución de inquietudes.

- Implantación del Programa Bilingüe en Educación Infantil con la finalidad de aprovechar el potencial que poseen los niños en esas edades o, en su defecto, aumento del número de horas de lengua extranjera inglés en esta etapa, para que el paso de los niños de esta etapa a Educación Primaria no sea tan significativo como lo está siendo actualmente. A este respecto, una entrevistada considera que sería adecuado que la maestra especialista en inglés que imparta la asignatura de lengua extranjera en Educación Infantil, concretamente a los niños de cinco años, les imparta docencia bilingüe en primero de Educación Primaria.

- Concesión de prioridad a los maestros implicados en estos programas para la realización de cursos de formación y en la solicitud de becas al extranjero.

En cuanto a la coordinación del profesorado, algunas de las propuestas que sugerimos son las siguientes:

- Definición y concreción a nivel legal de tiempos para las tareas de coordinación, así como una reducción horaria debido a la carga de trabajo.

- Mejora del proceso de selección de los auxiliares de conversación, incluyendo como uno de los criterios poseer titulaciones vinculadas con la educación, con la finalidad de que estén interesados por la enseñanza, implicándose y participando activamente en las aulas.

- Incremento de las horas de los auxiliares de conversación, puesto que doce horas por centro bilingüe no parecen suficientes.

Respecto a la enseñanza y Programa Bilingüe, una línea de acción sería:

- Establecimiento de un sistema de evaluación específico entre todos los docentes adscritos al programa, incorporando aquellos criterios que consideren imprescindibles valorar. 
En relación a los recursos e incentivos, algunas de las propuestas son:

- Aumento de recursos específicos y tecnológicos en estos centros, tales como libros en inglés, pizarras digitales, auriculares y ordenadores, así como un aula bilingüe, como mínimo, por colegio.

- Incremento de apoyos en las asignaturas impartidas en lengua inglesa destinados al alumnado con NEE e inmigrantes, con la finalidad de atender a la diversidad y ofrecer una educación de calidad para todos.

- Reconocimiento por participar en el programa.

Además, todos los maestros entrevistados proponen que no se convoquen más colegios bilingües hasta que no doten de medios suficientes a los centros docentes de las anteriores promociones, puesto que consideran una incoherencia incorporar más colegios al Programa Bilingüe sin garantizarles una educación bilingüe de calidad.

Por último, todos los profesores consideran que la Consejería debería de implicarse más en esta iniciativa puesto que no perciben su apoyo ante este nuevo reto educativo. Por ello, consideramos que sería recomendable escuchar a todos los docentes implicados en este programa, con la finalidad de responder a sus necesidades y evitar que, sumidos por su descontento, lo abandonen.

\section{REFERENCIAS BIBLIOGRÁFICAS}

Agar, M. H. (1980). The professional stranger: An informal introduction to ethnography. Nueva York: Academic Press.

Baker, C. (1997). Fundamentos de educación bilingüe y bilingüismo. Madrid: Cátedra.

Cancelas, L. P. y Cancelas, M. A. (2009). "AICLE: estableciendo las bases para trabajar la música en L2". TAVIRA 25: 137-159, disponible en: http://rodin.uca.es:8081/xmlui/bitstream/ handle/10498/10704/34914857.pdf? sequence=1, consultado el 27 de Octubre de 2012.

Castro, R. (2002). Bilingual Education: A reference Handbook. Santa Bárbara: ABC-CLIO.

Cenoz, J. and Jessner, U. (2000). English in Europe: The Acquisition of a Third Language. Clevendon: Multilingual Matters.

Cummins, J. (2002). Lenguaje, poder y pedagogía: niños y niñas bilingües entre dos fuegos. Madrid: Morata.

Corrales, K. (2009). "Construyendo un segundo idioma: El constructivismo y la enseñanza del L2" en Zona próxima: Revista del Instituto de Estudios en Educación Universidad del Norte, 10: 156-67.

Daniel, A. and Nerlich, B. (1998). "Analysing vocabulary acquisition in the Schleswig-Holstein immersion program", in J. Arnaud and J. M. Artigal (eds.), Immersion programs: A European perspective. Barcelona: Universidad de Barcelona, 647-657.

Fehling, S. (2002). "Methodische Überlegungen zur Erforschung von Language Awareness", in S. Breidbach, G. Bach and D. Wolff (eds.), Bilingualer Sachfachunterricht: Didaktik, Lehrer-/Lernerforschung und Bildungspolitik zwischen Theorie und Empirie. Frankfurt: Peter Lang, 161-172.

Fernández, J. G. (2008). "La Educación Física bilingüe en la etapa de Primaria: Descripción de una experiencia". Revista Digital 117: 1-1, disponible en: http://www.efdeportes.com/efd117/ la-educacion-fisica-bilingue-en-primaria.htm, consultado el 26 de Noviembre de 2012. 
Fernández, E. (2009). "La secuenciación de contenidos lingüísticos dentro de la Educación Física bilingüe". Revista Digital Innovación y Experiencias Educativas 23: 1-9, disponible en: http://www.csi-csif.es/andalucia/modules/mod_ense/revista/pdf/Numero_23/ELISEO_ FERNANDEZ 2.pdf, consultado el 1 de Diciembre de 2012.

Fernández, R., Pena, C., García, A. y Halbach, A. (2005):"La implantación de proyectos educativos bilingües en la Comunidad de Madrid: las expectativas del profesorado antes de iniciar el proyecto". Porta Linguarum 3: 161-173, disponible en: http://www.ugr.es/local/portalin, consultado el 18 de Septiembre de 2012.

Fernández, R. F. (2009). "Fomento y tratamiento del plurilingüismo en el área de Educación Física en Primaria". Emásf, revista digital de Educación Física 1: 1-9, disponible en: http://emasf.webcindario.com/, consultado el 20 de Noviembre de 2012.

García, J. V; García, J. J. y Yuste, J. L. (2012). "Educación Física en inglés: Una propuesta para trabajar la higiene postural en Educación Primaria". RETOS. Nuevas tendencias en Educación Física, Deporte y Recreación 22: 70-75, disponible en: http://dialnet.unirioja. es/servlet/articulo?codigo=3985146, consultado el 29 de Noviembre de 2012 .

González, A. (2005). "La Didáctica de la pragmática en el proceso enseñanza-aprendizaje de lenguas segundas y extranjeras: Algunas consideraciones". Lenguaje y textos 23: 127-138, disponible en: http://dialnet.unirioja.es/, consultado el 15 de Junio de 2012.

Halbach, A. (2008). "Una metodología para la enseñanza bilingüe en la etapa de Primaria". Revista de Educación 346: 455-66, disponible en: www.revistaeducacion.mec.es, consultado el 5 de Septiembre de 2012.

Howard, E. and Christian, D. (1997). The development of bilingualism and biliteracy in two way immersion students. Paper presented at the annual meeting of the American Educational Research Association. Washington: Educational Resources Information Center.

Howard, E., Christian, D. and Genesee, F. (2004). The development of bilingualism and biliteracy from grade 3 to 5: A summary of findings from the CAL/CREDE study of two way immersion education. Santa Cruz: Crede.

Howard, E., Sugarman, J. and Christian, D. (2003). Trends in two-way immersion education: A review of the research. Washington: Center for Applied Linguistics.

Krück, B. and Loeser, K. (eds.)(2002). Innovationen im Fremdsprachenunterricht 2: Fremdsprachen als Arbeittsprachen. Frankfurt: Peter Lang.

Lasagabaster, D. (2001). "Bilingualism, immersion programmes and language learning in the Basque Country", en Journal of Multilingual and Multicultural Development, 22, 5: 401-25.

Laorden, C. y Peñafiel, E. (2010). "Proyectos bilingües en los centros de la Comunidad Autónoma de Madrid: Percepción de los equipos directivos". Revista de Investigación Educativa 28, 2: 325-344, disponible en: http://revistas.um.es/rie, consultado el 25 de Julio de 2012.

Lorenzo, F., Casal, S., Moore, P. y Alfonso, Y. M. (2009). Bilingüismo y Educación: Situación de la Red de Centros Bilingües en Andalucía. Sevilla: Fundación Centro de Estudios Andaluces.

Molero, J. J. (2011a). "De la Educación Física hacia la Educación Física bilingüe: un análisis desde el punto de vista metodológico". Emásf, revista digital de Educación Física 10: 7-16, disponible en: http://emasf.webcindario.com/, consultado el 10 de septiembre de 2012.

Molero, J. J. (2011b). "La planificación de la Educación Física bilingüe: aspectos a tener en cuenta en la integración de los contenidos lingüísticos". Emásf, revista digital de Educación Física 9: 6-15, disponible en: http://emasf.webcindario.com/, consultado el 30 de Noviembre de 2012.

Olaziregi, I. (1994). "Evaluación de una experiencia de inmersión en el País Vasco", en M. Siguán (ed.), Las Lenguas en la escuela. Barcelona: ICE/HORSORI, 43-60. 
Pavón, V. and Rubio, F. (2010). "Teacher' s Concerns and Uncertainties about the Introduction of CLIL Programmes". Porta Linguarum 14: 45-58, disponible en: http://www.ugr.es/ portalin/ articulos/PL_numero14/3\%20Teachers\%20Concerns\%20and\%20Uncertainties_V\%20 Pavon_F\%20Rubio.pdf, consultado el 28 de Noviembre de 2012.

Pérez, M. E. (2008). "La enseñanza del inglés: un antes y un después de la implantación de la enseñanza bilingüe", en Revista Aula de innovación educativa, 168: 17-20.

Ramos, F. (2007a). "Opiniones de los alumnos de un programa bilingüe andaluz sobre su programa y el bilingüismo". Revista Electrónica de Investigación Educativa 9, 2: 1-15, disponible en: http://dialnet.unirioja.es/servlet/articulo?codigo=2389019, consultado el 12 de Septiembre de 2012.

Ramos, F. (2007b). "Programas bilingües y formación de profesores en Andalucía". Revista Iberoamericana de Educación 44:133-146, disponible en: www.rieoei.org/, consultado el 11 de Septiembre de 2012.

Ramos, F. (2009). "Una propuesta de AICLE para el trabajo con textos en segundos idiomas". Porta Linguarum 12:169-182, disponible en: http://www.ugr.es/local/portalin, consultado el 28 de Julio de 2012.

Ramos, F. y Ruiz, J. V. (2011). "La Educación Física en centros bilingües inglés-español: de las singularidades propias del área a la elaboración de propuesta didácticas prácticas con AICLE". RESLA 24: 153-170, disponible en: http://dialnet.unirioja.es/servlet/articulo?codigo=3886031, consultado el 28 de Noviembre de 2012.

Rodríguez, G., Gil, J. y García, E. (1996). Metodología de la investigación cualitativa. Málaga: Aljibe.

Rodríguez, M. (2010). “Unidad didáctica bilingüe: Do you play tennis?”. Emásf, Revista digital de Educación física 5: 1-20, disponible en: http://emasf.webcindario.com/, consultado el 15 de Julio de 2012.

Ruiz de Zarobe, Y. (2005). "Age and third language production: A longitudinal study" en Internacional Journal of Multilingualism, 2, 2: 105-12.

Sánchez, M. P. y Rodríguez, R. (1986). "La educación bilingüe y el aprendizaje de una segunda lengua: sus características y principios fundamentales". Infancia y Aprendizaje 33: 3-26, disponible en: http://www.fia.es/revistas, consultado el 2 de Septiembre de 2012.

Siguán, M. y Mackey, W. F. (1986). Educación y Bilingüismo. Madrid: Santillana.

Siguán, M. (1992). España Plurilingüe. Madrid: Alianza.

Soltero, S. W. (2004). Dual Language: teaching and Learning in two Languages. Boston: Allyn and Bacon.

Tójar, J. C. (2006). Investigación cualitativa: comprender y actuar. Madrid: La Muralla.

Torres-Guzmán, M. and Etxebarria, F. (2005). "Modelo B/Dual language programmes in the Basque Country and the USA", in Internacional Journal of Bilingual Education and Bilingualism, 8, 6: 506-28.

Travé, G. (2013). "Un estudio sobre las representaciones del profesorado de Educación Primaria acerca de la enseñanza bilingüe". Revista de Educación 361: 1-14, disponible en: http:// www.revistaeducacion.mec.es/doi/361_149.pdf, consultado el 29 de Noviembre de 2012.

Trujillo, F. (2007). "Enseñar nuevas lenguas en la escuela: L1, L2, LE..., NL". Revista de Educación 343: 71-91, disponible en: www.revistaeducacion.mec.es, consultado el 16 de Septiembre de 2012.

Urrutia, H., Cantia, L., Martínez, M. D. y Millas, F. (1998). Bilingüismo y rendimiento académico en la Comunidad Autónoma Vasca. Bilbao: Jóvenes por la Paz. 
Zydatiss, W. (2002). "Die Erfassung der interaktiven Gesprächsfähigkeit mithilfe eines Kommunikativen Tests: Holistiche vs. analytische und externe vs. objektivierte Bewertung”, in B. Krück und K. Loeser (eds.), Innovationen im Fremdsprachenunterricht 2: Fremdsprachen als Arbeittsprachen. Frankfurt: Peter Lang, 153-174. 